\title{
Local stability of energy estimates for the Navier-Stokes equations.
}

\author{
Diego Chamorro, Pierre Gilles Lemarié-Rieusset, and Kawther Mayoufi
}

\begin{abstract}
We study the regularity of the weak limit of a sequence of dissipative solutions to the Navier-Stokes equations when no assumptions is made on the behavior of the pressures.
\end{abstract}

\section{Local weak solutions.}

In this paper, we are interested in local properties (regularity, local energy estimates) of weak solutions of Navier-Stokes equations.

Definition 1.1 (Local weak solutions). Let $\Omega$ be a domain in $\mathbb{R} \times \mathbb{R}^{3}$ and $\vec{f} \in L_{\text {loc }}^{2}(\Omega)$ a divergence-free time-dependent vector field. A vector field $\vec{u}$ will be said to be a local weak solution of the Navier-Stokes equations on $\Omega$ (associated to the force $\vec{f}$ ) if, for each cylinder $Q=I \times O$ (where $I$ is an open interval in $\mathbb{R}$ and $O$ an open subset of $\mathbb{R}^{3}$ ) such that $\bar{Q}$ is a compact subset of $\Omega$, we have $\vec{u} \in L_{t}^{\infty} L_{x}^{2}(Q) \cap L_{t}^{2} H_{x}^{1}(Q), \vec{u}$ is divergence-free and, for every smooth compactly supported divergence-free vector field $\vec{\phi} \in \mathcal{D}(Q)$ we have

$$
\iint_{Q} \vec{u} \cdot\left(\partial_{t} \vec{\phi}+\Delta \vec{\phi}\right)+\vec{u} \cdot(\vec{u} \cdot \vec{\nabla} \vec{\phi})+\vec{f} \cdot \vec{\phi} d t d x=0
$$

More precisely, we shall address the behavior of a weak limit of regular solutions.

DEFINITION 1.2 (Regular local solutions). Let $\Omega$ be a domain in $\mathbb{R} \times \mathbb{R}^{3}$ and $\vec{f} \in L_{\text {loc }}^{2}(\Omega)$ a divergence-free time-dependent vector field and $\vec{u}$ a local weak solution of the Navier-Stokes equations on $\Omega$ (associated to the force $\vec{f}$ ).

A) $\vec{u}$ is a regular local solution if, for each cylinder $Q \subset \subset \Omega$, we have $\vec{u} \in$ $L_{t, x}^{\infty}(Q)$

B) The set $R(\vec{u})$ of regular points of $\vec{u}$ is the largest open subset of $\Omega$ on which $\vec{u}$ is a regular solution. The set $\Sigma(\vec{u})$ of singular points is the complement of $R(\vec{u}): \Sigma(\vec{u})=\Omega \backslash R(\vec{u})$.

Our result is then the following one $[\mathbf{M}]$ :

1991 Mathematics Subject Classification. Primary 35Q30, 76D05.

Key words and phrases. Navier-Stokes equations; Partial regularity; Caffarelli, Kohn and Nirenberg theory; Local energy inequalities. 
THEOREM 1.3 (Singular points of a weak limit.). Let $\Omega$ be a domain in $\mathbb{R} \times \mathbb{R}^{3}$. Assume that we have sequences $\vec{f}_{n}$ of divergence-free time-dependent vector fields and $\vec{u}_{n}$ of local weak solutions of the Navier-Stokes equations on $\Omega$ (associated to the forces $\vec{f}_{n}$ ) such that, for each cylinder $Q \subset \subset \Omega$, we have

- $\vec{f}_{n} \in L_{t}^{2} H_{x}^{1}(Q)$ and $\vec{f}_{n}$ converges weakly in $L_{t}^{2} H_{x}^{1}$ to a limit $\vec{f}$,

- the sequence $\vec{u}_{n}$ is bounded in $L_{t}^{\infty} L_{x}^{2}(Q) \cap L_{t}^{2} H_{x}^{1}(Q)$ and converges weakly in $L_{t}^{2} H_{x}^{1}(Q)$ to a limit $\vec{u}$,

- for every $n, \vec{u}_{n}$ is bounded on $Q$.

Then the limit $\vec{u}$ is a local weak solution on $\Omega$ of the Navier-Stokes equations associated to the force $\vec{f}$, and its set $\Sigma(\vec{u})$ has parabolic one-dimensional Hausdorff measure equal to 0.

As we shall see, the main tool of the proof is an extension of the CaffarelliKohn-Nirenberg theory [Ca to the case where we have no control on the pressure (i.e. the case of generalized suitable solutions $[\mathbf{W}]$ or dissipative solutions $[\mathbf{C h}]$ ).

\section{Pressure.}

Equations (10) can classically be rewritten as an equation involving a pressure term. See for instance $[\mathbf{W}$. In the following, we shall only need the pressure inside spherical cylinders $Q=I \times B$ (where $I$ is an open interval in $\mathbb{R}$ and $B$ an open ball of $\mathbb{R}^{3}$ ). In that case, it is very easy to define a pressure $p$ such that

$$
\partial_{t} \vec{u}=\Delta \vec{u}-\vec{u} \cdot \vec{\nabla} \vec{u}-\vec{\nabla} p+\vec{f} \quad \text { in } \mathcal{D}^{\prime}(Q) .
$$

Indeed, let $Q, Q^{\#}$, and $Q^{*}$ be three relatively compact cylinders in $\Omega$ with $\bar{Q} \subset Q^{\#}$ and $\overline{Q^{\#}} \subset Q^{*}$ and $\psi$ a cut off smooth function supported in $Q^{*}$ and equal to 1 on a neighboorhood of $\overline{Q^{\#}}$. The function

$$
p_{0}=-\frac{1}{\Delta}\left(\sum_{i=1}^{3} \sum_{j=1}^{3} \partial_{i} \partial_{j}\left(\psi u_{i} u_{j}\right)\right)
$$

belongs to $L_{t}^{2} L_{x}^{3 / 2}$ and, on $Q^{\#}$, the distribution

$$
\vec{T}=\partial_{t} \vec{u}-\Delta \vec{u}+\vec{u} \cdot \vec{\nabla} \vec{u}+\vec{\nabla} p_{0}-\vec{f}
$$

satisfies

$$
\text { curl } \vec{T}=0 \text { and } \operatorname{div} \vec{T}=0 .
$$

Moreover, $\vec{T}_{0}=\vec{T}-\partial_{t} \vec{u}$ belongs to $L_{t}^{2} H_{x}^{-2}\left(Q^{\#}\right)$. Picking $t_{0} \in I$, we define $\vec{S}=$ $\vec{u}+\int_{t_{0}}^{t} \vec{T}_{0}(s,) d$.$s . We have \vec{S} \in L_{t}^{\infty} H_{x}^{-2}\left(Q^{\#}\right)$. Moreover, we have $\partial_{t} \operatorname{curl} \vec{S}=0$ and $\partial_{t} \operatorname{div} \vec{S}=0$. Thus, if $\alpha \in \mathcal{D}(I)$ with $\int \alpha d t=1$, we find that

$$
\vec{S}_{0}=\vec{S}-\int_{I} \alpha(s) \vec{S}(s, .) d s
$$

satisfies

$$
\partial_{t} \vec{S}_{0}=\vec{T}, \quad \text { curl } \vec{S}_{0}=0 \text { and } \operatorname{div} \vec{S}_{0}=0
$$

In particular,

$$
\Delta \vec{S}_{0}=\vec{\nabla}\left(\operatorname{div} \vec{S}_{0}\right)-\vec{\nabla} \wedge\left(\operatorname{curl} \vec{S}_{0}\right)=0 .
$$


Thus, we get that $\vec{S}_{0}$ is smooth in the space variable; in particular $\vec{S}_{0} \in L_{t}^{\infty} W_{x}^{1, \infty}(Q)$. If $x_{0} \in B$ and if we define

$$
\varpi(t, x)=\int_{0}^{1} \vec{S}_{0}\left(t,(1-\theta) x_{0}+\theta x\right) \cdot\left(x-x_{0}\right) d \theta,
$$

we find that $\varpi \in L_{t, x}^{\infty}(Q)$ and $\vec{\nabla} \varpi=\vec{S}_{0}$. Defining $p=p_{0}-\partial_{t} \varpi$, we find the equality (2).

Of course, the pressure may be singular in time (as $\partial_{t} \varpi$ is only the derivative of a bounded function). We shall comment further on this in Sections 3 and 5

\section{Energy balance.}

This section is devoted to the study of $\partial_{t}|\vec{u}|^{2}$, as it is the main tool to estimate the partial regularity of $\vec{u}$. If $\vec{u}$ and the pressure $p$ were regular, we could write from equality (2)

$$
\partial_{t}|\vec{u}|^{2}=2 \vec{u} \cdot \partial_{t} \vec{u}=2 \vec{u} \cdot \Delta \vec{u}-2 \vec{u} \cdot(\vec{u} \cdot \vec{\nabla} \vec{u}+\vec{\nabla} p)+2 \vec{u} \cdot \vec{f}
$$

and rewrite

and, since $\operatorname{div} \vec{u}=0$,

$$
2 \vec{u} \cdot \Delta \vec{u}=\Delta\left(|\vec{u}|^{2}\right)-2|\vec{\nabla} \otimes \vec{u}|^{2}
$$

$$
2 \vec{u} \cdot(\vec{u} \cdot \vec{\nabla} \vec{u}+\vec{\nabla} p)=\operatorname{div}\left(\left(|\vec{u}|^{2}+2 p\right) \vec{u}\right) .
$$

This would give the following local energy balance in $Q$

$$
\partial_{t}|\vec{u}|^{2}=\Delta\left(|\vec{u}|^{2}\right)-2|\vec{\nabla} \otimes \vec{u}|^{2}-\operatorname{div}\left(\left(|\vec{u}|^{2}+2 p\right) \vec{u}\right)+2 \vec{u} \cdot \vec{f} .
$$

However, local weak solutions (and their associates pressures) are not regular enough to allow those computations : the problem lies in the fact that the terms $\vec{u} \cdot(\vec{u} \cdot \nabla \vec{u})$ and $\vec{u} \cdot \vec{\nabla} p$ are not well defined in $\mathcal{D}^{\prime}$. If the pressure is regular enough (for instance, $p \in L_{t, x}^{3 / 2}(Q)$ ) then one first smoothens $\vec{u}$ with a mollifier $\varphi_{\epsilon}=\frac{1}{\epsilon^{3}} \varphi\left(\frac{x}{\epsilon}\right)$, defining $\vec{u}_{\epsilon}=\varphi_{\epsilon} * \vec{u}$. One then finds

$\partial_{t}\left|\vec{u}_{\epsilon}\right|^{2}=\Delta\left(\left|\vec{u}_{\epsilon}\right|^{2}\right)-2\left|\vec{\nabla} \otimes \vec{u}_{\epsilon}\right|^{2}-2 \vec{u}_{\epsilon} \cdot \varphi_{\epsilon} *(\vec{u} . \vec{\nabla} \vec{u})-2 \operatorname{div}\left(\left(p * \varphi_{\epsilon}\right) \vec{u}_{\epsilon}\right)+2 \vec{u}_{\epsilon} \cdot\left(\varphi_{\epsilon} * \vec{f}\right)$.

The limit $\epsilon \rightarrow 0$ gives then

$$
\partial_{t}|\vec{u}|^{2}=\Delta\left(|\vec{u}|^{2}\right)-2|\vec{\nabla} \otimes \vec{u}|^{2}-2 \lim _{\epsilon \rightarrow 0} \vec{u}_{\epsilon} \cdot \varphi_{\epsilon} *(\vec{u} \cdot \vec{\nabla} \vec{u})-2 \operatorname{div}(p \vec{u})+2 \vec{u} \cdot \vec{f} .
$$

In order to compare this expression with (3), we define

$$
M_{\epsilon}(\vec{u})=-\operatorname{div}\left(|\vec{u}|^{2} \vec{u}\right)+2 \vec{u}_{\epsilon} \cdot \varphi_{\epsilon} *(\vec{u} \cdot \vec{\nabla} \vec{u})
$$

and write

$$
\partial_{t}|\vec{u}|^{2}=\Delta\left(|\vec{u}|^{2}\right)-2|\vec{\nabla} \otimes \vec{u}|^{2}-\operatorname{div}\left(\left(|\vec{u}|^{2}+2 p\right) \vec{u}\right)+2 \vec{u} \cdot \vec{f}-\lim _{\epsilon \rightarrow 0} M_{\epsilon}(\vec{u}) .
$$

However, our assumptions on weak solutions don't allow us to make all those computations, as the pressure we can define on $Q$ has no regularity with respect to the time variable, so that $p \vec{u}$ is not well defined in $\mathcal{D}^{\prime}$. Thus, one must smoothens as well $\vec{u}$ with respect to the time variable, with a mollifier $\psi_{\eta}(t)=\frac{1}{\eta} \psi\left(\frac{t}{\eta}\right)$. Defining $\vec{u}_{\epsilon, \eta}=\psi_{\eta} *_{t} \varphi_{\epsilon} *_{x} \vec{u}=\xi_{\eta, \epsilon} *_{t, x} \vec{u}$, one finds

$$
\begin{aligned}
\partial_{t}\left|\vec{u}_{\epsilon, \eta}\right|^{2}= & \Delta\left(\left|\vec{u}_{\epsilon, \eta}\right|^{2}\right)-2\left|\vec{\nabla} \otimes \vec{u}_{\epsilon, \eta}\right|^{2}-2 \vec{u}_{\epsilon, \eta} \cdot \xi_{\eta, \epsilon} *(\vec{u} \cdot \vec{\nabla} \vec{u}) \\
& -2 \operatorname{div}\left(\left(p * \xi_{\eta, \epsilon}\right) \vec{u}_{\epsilon, \eta}\right)+2 \vec{u}_{\epsilon, \eta} \cdot\left(\xi_{\eta, \epsilon} * \vec{f}\right) .
\end{aligned}
$$


The limit $\eta \rightarrow 0$ gives then

$\partial_{t}\left|\vec{u}_{\epsilon}\right|^{2}=\Delta\left(\left|\vec{u}_{\epsilon}\right|^{2}\right)-2\left|\vec{\nabla} \otimes \vec{u}_{\epsilon}\right|^{2}-2 \vec{u}_{\epsilon} \cdot \varphi_{\epsilon} *(\vec{u} \cdot \vec{\nabla} \vec{u})-2 \lim _{\eta \rightarrow 0} \operatorname{div}\left(\left(p * \xi_{\eta, \epsilon}\right) \vec{u}_{\epsilon, \eta}\right)+2 \vec{u}_{\epsilon} \cdot\left(\varphi_{\epsilon} * \vec{f}\right)$.

The limit $\epsilon \rightarrow 0$ gives finally

$$
\begin{aligned}
\partial_{t}|\vec{u}|^{2} & =\Delta\left(|\vec{u}|^{2}\right)-2|\vec{\nabla} \otimes \vec{u}|^{2} \\
& -2 \lim _{\epsilon \rightarrow 0}\left(\vec{u}_{\epsilon} \cdot \varphi_{\epsilon} *(\vec{u} \cdot \vec{\nabla} \vec{u})+\lim _{\eta \rightarrow 0} \operatorname{div}\left(\left(p * \xi_{\eta, \epsilon}\right) \vec{u}_{\epsilon, \eta}\right)\right)+2 \vec{u} \cdot \vec{f} .
\end{aligned}
$$

In order to circumvene the problems of lack of regularity for the pressure, we introduce the notion of harmonic correction :

DEFINITION 3.1 (Harmonic corrections). Let $\Omega$ be a domain in $\mathbb{R} \times \mathbb{R}^{3}, \vec{f} \in$ $L_{\text {loc }}^{2}(\Omega)$ a divergence-free time-dependent vector field and $\vec{u}$ a local weak solution of the Navier-Stokes equations on $\Omega$ (associated to the force $\vec{f}$ ). A harmonic correction $\vec{H}$ on a cylinder $Q \subset \subset \Omega$ is a vector field such that

- $\operatorname{div} \vec{H}=0$ and $\Delta \vec{H}=0$,

- $\vec{H} \in L_{t, x}^{\infty}(Q)$ and $\partial_{i} \vec{H} \in L_{t, x}^{\infty}(Q)$ for $i=1,2,3$,

- there exists $\vec{F} \in L_{t, x}^{2}(Q)$ and $P \in L_{t, x}^{3 / 2}(Q)$ such that the vector field $\vec{U}=\vec{u}+\vec{H}$ satisfies

$$
\partial_{t} \vec{U}=\Delta \vec{U}-\vec{U} \cdot \vec{\nabla} \vec{U}-\vec{\nabla} P+\vec{F} .
$$

In the literature, one can find at least two such harmonic corrections for local weak solutions :

Lemma 3.2. Let $\Omega$ be a domain in $\mathbb{R} \times \mathbb{R}^{3}, \vec{f} \in L_{\text {loc }}^{2}(\Omega)$ a divergence-free timedependent vector field and $\vec{u}$ a local weak solution of the Navier-Stokes equations on $\Omega$ (associated to the force $\vec{f}$ ). Let $Q$ be a spherical cylinder in $\Omega$. Then:

A) the decomposition of the pressure $p$ as $p=p_{0}-\partial_{t} \varpi$ described in Section 1 provides a harmonic correction $\vec{H}=-\vec{\nabla} \varpi$ of $\vec{u}$ on $Q$,

B) Let $\psi(t, x)=\alpha(t) \beta(x)$ be a smooth cut-off function supported by a cylinder $Q^{*} \subset \subset \Omega$ and equal to 1 on a neighborhood of $Q$. Then $\vec{U}=-\frac{1}{\Delta} \vec{\nabla} \wedge(\psi \vec{\nabla} \vec{u})$ is such that $\vec{H}=\vec{U}-\vec{u}$ is a harmonic correction of $\vec{u}$ on $Q$.

Proof. The case of $\vec{H}=-\vec{\nabla} \varpi$ has been discussed by Wolf $\mathbf{W}$. For $\vec{U}=$ $\vec{u}-\vec{\nabla} \varpi$, we have $\vec{\nabla} \wedge \vec{U}=\vec{\nabla} \wedge \vec{u}$ and $\Delta \vec{U}=\Delta \vec{u}$, so that

$$
\begin{aligned}
\partial_{t} \vec{U}-\Delta \vec{U}+\vec{U} \cdot \vec{\nabla} \vec{U} & =\partial_{t} \vec{u}-\partial_{t} \vec{\nabla} \varpi-\Delta \vec{u}+(\vec{\nabla} \wedge \vec{u}) \wedge(\vec{u}-\vec{\nabla} \varpi)+\vec{\nabla}\left(\frac{|\vec{U}|^{2}}{2}\right) \\
& =\vec{\nabla}\left(\frac{|\vec{U}|^{2}}{2}-\frac{|\vec{u}|^{2}}{2}-p_{0}\right)+\vec{f}-(\vec{\nabla} \wedge \vec{u}) \wedge \vec{\nabla} \varpi
\end{aligned}
$$

We may then decompose $(\vec{\nabla} \wedge \vec{u}) \wedge \vec{\nabla} \varpi \in L_{t}^{2} L_{x}^{2}(Q)$ into $\vec{f}_{1}+\vec{\nabla} p_{1}$ with $\vec{f}_{1} \in L_{t}^{2} L_{x}^{2}$ and $\operatorname{div} \vec{f}_{1}=0$ and $p_{1} \in L_{t}^{2} L_{x}^{6}(Q)$ (for instance, by extending $(\vec{\nabla} \wedge \vec{u}) \wedge \vec{\nabla} \varpi$ by 0 outside $Q$ and then using the Leray projection operator). We thus find

$$
P=p_{0}+\frac{|\vec{u}|^{2}}{2}-\frac{|\vec{U}|^{2}}{2}+p_{1} \text { and } \vec{F}=\vec{f}-\vec{f}_{1} \text {. }
$$


The case of $\vec{U}=-\frac{1}{\Delta} \vec{\nabla} \wedge(\psi \vec{\nabla} \vec{u})$ has been discussed by Chamorro, LemariéRieusset and Mayoufi in $[\mathbf{C h}, \mathbf{L e}$. It is worth noticing that the pressure $P$ they obtain belongs to $L_{t}^{2} L_{x}^{q}(Q)$ for every $q<3 / 2$.

Note that, in both cases, even if $\vec{f}$ is assumed to be more regular, we cannot get a better regularity for $\vec{F}$ than $L_{t}^{2} L_{x}^{2}$, because of the contribution of $(\vec{\nabla} \wedge \vec{u}) \wedge \vec{H}$ to the force.

An important result of Chamorro, Lemarié-Rieusset and Mayoufi is the following one $\mathbf{C h}, \mathbf{L e}$ :

Theorem 3.3 (Energy balance). Let $\Omega$ be a domain in $\mathbb{R} \times \mathbb{R}^{3}, \vec{f} \in L_{\text {loc }}^{2}(\Omega)$ a divergence-free time-dependent vector field and $\vec{u}$ a local weak solution of the Navier-Stokes equations on $\Omega$ (associated to the force $\vec{f}$ ). Let $Q$ be a spherical cylinder in $\Omega$ and $p$ the pressure associated to $\vec{u}$ on $Q$. Then:

A) The quantities

$$
M(\vec{u})=\lim _{\epsilon \rightarrow 0}\left(-\operatorname{div}\left(|\vec{u}|^{2} \vec{u}\right)+2 \vec{u}_{\epsilon} \cdot \varphi_{\epsilon} *(\vec{u} \cdot \vec{\nabla} \vec{u})\right)
$$

and

$$
<<\operatorname{div}(p \vec{u})>>=\lim _{\epsilon \rightarrow 0} \lim _{\eta \rightarrow 0} \operatorname{div}\left(\left(p * \xi_{\eta, \epsilon}\right) \vec{u}_{\epsilon, \eta}\right)
$$

are well defined in $\mathcal{D}^{\prime}(Q)$.

B) We have the energy balance on $Q$ :

$$
\partial_{t}|\vec{u}|^{2}=\Delta\left(|\vec{u}|^{2}\right)-2|\vec{\nabla} \otimes \vec{u}|^{2}-\operatorname{div}\left(|\vec{u}|^{2} \vec{u}\right)-2<<\operatorname{div}(p \vec{u})>>+2 \vec{u} \cdot \vec{f}-M(\vec{u}) .
$$

C) $M(\vec{u})$ can be computed as a defect of regularity. More precisely, we have, for

$A_{k, \epsilon}(\vec{u})=\frac{\left(u_{k}(t, x-y)-u_{k}(t, x)\right)(\vec{u}(t, x-y)-\vec{u}(t, x)) \cdot \int \varphi_{\epsilon}(z)(\vec{u}(t, x-z)-\vec{u}(t, x)) d z}{\epsilon}$ and

$$
B_{k, \epsilon}(\vec{u})=\frac{\left(u_{k}(t, x-y)-u_{k}(t, x)\right)|\vec{u}(t, x-y)-\vec{u}(t, x)|^{2}}{\epsilon},
$$

the identity

$$
M_{\epsilon}(\vec{u})=\sum_{k=1}^{3} \int \frac{1}{\epsilon^{3}} \partial_{k} \varphi\left(\frac{y}{\epsilon}\right)\left(2 A_{k, \epsilon}(\vec{u})-B_{k, \epsilon}(\vec{u})\right) d y-C_{\epsilon}(\vec{u})
$$

where $\lim _{\epsilon \rightarrow 0} C_{\epsilon}(\vec{u})=0$ in $\mathcal{D}^{\prime}(Q)$.

D) If $\vec{U}=\vec{u}+\vec{H}$ where $\vec{H}$ is a harmonic correction of $\vec{u}$, then $M(\vec{U})=M(\vec{u})$.

Proof. The key tool is identity (5) which has been described by Duchon and Robert $\mathbf{D}$ for any divergence-free vector field $\vec{u}$ in $L_{t}^{\infty} L_{x}^{2}(Q) \cap L_{t}^{2} H_{x}^{1}(Q)$. Let us remark that if $w_{1}$ and $w_{2}$ belong to $L_{t}^{\infty} L_{x}^{2}(Q) \cap L_{t}^{2} H_{x}^{1}(Q)$ and $w_{3}$ to $L_{t}^{\infty} \operatorname{Lip}_{x}(Q)$ then we have obviously

$\lim _{\epsilon \rightarrow 0} \int \frac{1}{\epsilon^{3}} \partial_{k} \varphi\left(\frac{y}{\epsilon}\right) \frac{\left(w_{1}(x-y)-w_{1}(x)\right)\left(w_{2}(x-y)-w_{2}(x)\right)\left(w_{3}(x-y)-w_{3}(x)\right)}{\epsilon} d y=0$.

Thus, if $\vec{H}$ is a harmonic correction of $\vec{u}$, we have $\lim _{\epsilon \rightarrow 0} M_{\epsilon}(\vec{u}+\vec{H})-M_{\epsilon}(\vec{u})=0$. Since the $\operatorname{limits}_{\lim _{\epsilon \rightarrow 0}} M_{\epsilon}(\vec{u}+\vec{H})$ and $\lim _{\epsilon \rightarrow 0}\left(M_{\epsilon}(\vec{u})+2 \lim _{\eta \rightarrow 0} \operatorname{div}\left(\left(p * \xi_{\eta, \epsilon}\right) \vec{u}_{\epsilon, \eta}\right)\right)$ 
are well defined in $\mathcal{D}^{\prime}(Q)$, we find that $M(\vec{u})$ and $<<\operatorname{div}(p \vec{u})>>$ are well defined and that $M(\vec{u})=M(\vec{u}+\vec{H})$.

Of course, if $\vec{u}$ is regular enough, we have $M(\vec{u})=0$. Due to formula (5), Duchon and Robert [D] could see that when $\vec{u}$ belongs locally to $L_{t}^{3}\left(B_{3, q}^{1 / 3}\right)_{x}$ with $q<+\infty$, then $M(\vec{u})=0$. This is the case when the classical criterion $\vec{u} \in L_{t, x}^{4}(\Omega)$ is fulfilled, since $L_{t}^{4} L_{x}^{4} \cap L_{t}^{2} H_{x}^{1} \subset L_{t}^{3}\left(B_{3,3}^{1 / 3}\right)_{x}$. In particular, the support of the distribution $M(\vec{u})$ is a subset of the set $\Sigma(\vec{u})$ of singular points.

\section{Dissipativity and partial regularity.}

The best result we know about (partial) regularity of weak solutions has been given in 1982 by Caffarelli, Kohn and Nirenberg [Ca, $\mathbf{L a}$. Their result is based on the notion of suitable solutions (due to Scheffer $[\mathbf{S c}]$ ):

DEFINITION 4.1 (Suitable solutions). Let $\vec{u}$ be a local weak solutions of the Navier-Stokes solutions on a domain $\Omega \subset \mathbb{R} \times \mathbb{R}^{3}$. Then $\vec{u}$ is suitable if if satisfies the following two conditions :

- the pressure $p$ is locally in $L_{t, x}^{3 / 2}$,

- $M(\vec{u}) \geq 0$ (i.e. $M(\vec{u})$ is a non-negative locally finite Borel measure).

Let us define now the parabolic metric $\rho((t, x),(s, y))=\max \left(\sqrt{|t-s|},|x-y|^{2}\right)$ and the parabolic cylinders $Q_{r}(t, x)=\{(s, y): \rho((t, x),(s, y))<r\}$.

Theorem 4.2 (Caffarelli, Kohn and Nirenberg's regularity theorem). Let $\Omega$ be a domain in $\mathbb{R} \times \mathbb{R}^{3}, \vec{f} \in L_{\text {loc }}^{2}(\Omega)$ a divergence-free time-dependent vector field and $\vec{u}$ a local weak solution of the Navier-Stokes equations on $\Omega$ (associated to the force $\vec{f})$. Assume that moreover

- $\vec{u}$ is suitable,

- the force $\vec{f}$ is regular : $\vec{f}$ belongs locally to $L_{t}^{2} H_{x}^{1}$,

Then:

- if $(t, x) \notin \Sigma(\vec{u})$, there exists a neighborhood of $(t, x)$ on which $\vec{u}$ is Hölderian (with respect to the parabolic metric $\rho$ ) and we have

$$
\lim _{r \rightarrow 0} \frac{1}{r} \iint_{Q_{r}(t, x)}|\vec{\nabla} \otimes \vec{u}|^{2} d s d y=0 .
$$

- if $(t, x) \in \Sigma(\vec{u})$, then

$$
\limsup _{r \rightarrow 0} \frac{1}{r} \iint_{Q_{r}(t, x)}|\vec{\nabla} \otimes \vec{u}|^{2} d s d y>\epsilon^{*},
$$

where $\epsilon^{*}$ is a positive constant (which doesn't depend on $\vec{u}, \vec{f}$ nor $\Omega$ ).

The size of $\Sigma(\vec{u})$ is then easily controlled with the following lemma :

Lemma 4.3 (Parabolic Hausdorff dimension.). Let $u$ belongs locally to $L_{t}^{2} H_{x}^{1}$ and let $\Sigma$ be the set defined by

$$
(t, x) \in \Sigma \Leftrightarrow \limsup _{r \rightarrow 0} \frac{1}{r} \iint_{Q_{r}(t, x)}|\vec{\nabla} u|^{2} d s d y>0 .
$$

Then $\Sigma$ has parabolic one-dimensional Hausdorff measure equal to 0. 
Chamorro, Lemarié-Rieusset and Mayoufi $\mathbf{C h}$ have considered the case where no integrability assumptions were made on the pressure $p$. This implies to change the definition of suitable solutions. Following [D], they introduced the notion of dissipative solutions :

Definition 4.4 (Dissipative solutions). Let $\vec{u}$ be a local weak solutions of the Navier-Stokes solutions on a domain $\Omega \subset \mathbb{R} \times \mathbb{R}^{3}$. Then $\vec{u}$ is dissipative if $M(\vec{u}) \geq 0$.

A similar notion has been given by Wolf $\mathbf{\mathbf { W }}$. Indeed, if $\vec{u}$ is dissipative and if we use the harmonic correction $\vec{H}=-\vec{\nabla} \varpi$, we find, for $\vec{U}=\vec{u}+\vec{H}$ :

$$
\begin{aligned}
M(\vec{U})= & -\partial_{t}|\vec{U}|^{2}+\Delta\left(|\vec{U}|^{2}\right)-2|\vec{\nabla} \otimes \vec{U}|^{2}-\operatorname{div}\left(|\vec{U}|^{2} \vec{U}\right)-2 \operatorname{div}(P \vec{U})+2 \vec{U} \cdot \vec{F} \\
= & -\partial_{t}|\vec{U}|^{2}+\Delta\left(|\vec{U}|^{2}\right)-2|\vec{\nabla} \otimes \vec{U}|^{2}-\operatorname{div}\left(\left(|\vec{U}|^{2}+2 p_{0}\right) \vec{U}\right) \\
& +2 \vec{U} \cdot \vec{f}-2 \vec{U} \cdot \vec{f}_{1}-2 \operatorname{div}\left(p_{1} \vec{U}\right) \\
= & -\partial_{t}|\vec{U}|^{2}+\Delta\left(|\vec{U}|^{2}\right)-2|\vec{\nabla} \otimes \vec{U}|^{2}-\operatorname{div}\left(\left(|\vec{U}|^{2}+2 p_{0}\right) \vec{U}\right) \\
& +2 \vec{U} \cdot \vec{f}+2 \vec{U} \cdot(\vec{\nabla} \varpi \wedge(\vec{\nabla} \wedge \vec{U})) .
\end{aligned}
$$

Writing $M(\vec{U}) \geq 0$ is exactly expressing that $\vec{u}$ is a generalized suitable solution, as defined by Wolf.

Another tool used by Chamorro, Lemarié-Rieusset and Mayoufi is the notion of parabolic Morey space :

Definition 4.5 (Parabolic Morrey spaces). A function $\theta$ belongs to the parabolic Morrey space $\mathcal{M}^{s, \tau}(\Omega)$ if

$$
\sup _{x_{0}, t_{0}, r} \frac{1}{r^{5\left(1-\frac{s}{\tau}\right)}} \iint_{\Omega} 1_{\left|t-t_{0}\right|<r^{2}} 1_{\left|x-x_{0}\right|<r}|\theta(t, x)|^{s} d t d x<+\infty .
$$

Parabolic Morrey spaces have been used by Kukavica $[\mathbf{K}]$ in a variant of Caffarelli, Kohn and Nirenberg's theorem $[\mathbf{C a}$, and by O'Leary $\mathbf{O}$, Le in a variant of Serrin's regularity theorem $[\mathbf{S e}]$ :

THEOREM 4.6 (Kukavica's theorem). There exists a positive constant $\epsilon^{*}$ such that the following holds : If $\vec{U}$ is a solution of the Navier-Stokes equations on a domain $\Omega_{1}$, associated to a force $\vec{F}$ and a pressure $P$ and if $x_{0}, t_{0}, \vec{U}, P$ and $\vec{F}$ satisfy the following assumptions

- $\vec{U}$ belongs to $L_{t}^{\infty} L_{x}^{2} \cap L_{t}^{2} H_{x}^{1}$,

- $P \in L_{t, x}^{3 / 2}(\Omega)$,

- $\operatorname{div} \vec{F}=0$ and $\vec{F} \in L_{t, x}^{2}\left(\Omega_{1}\right)$,

- $\vec{U}$ is suitable,

- $\left(t_{0}, x_{0}\right) \in \Omega_{1}$ and

$$
\limsup _{r \rightarrow 0} \frac{1}{r} \iint_{\left(t_{0}-r^{2}, t_{0}+r^{2}\right) \times B\left(x_{0}, r\right)}|\vec{\nabla} \otimes \vec{U}|^{2} d s d x<\epsilon^{*},
$$

then there exists $\tau>5$ and a neighborhood $\Omega_{2}$ of $\left(t_{0}, x_{0}\right)$ such that $\vec{U} \in \mathcal{M}^{3, \tau}\left(\Omega_{2}\right)$.

TheOREM 4.7 (O'Leary's theorem). If $\vec{u}$ is a solution of the Navier-Stokes equations on a domain $\Omega_{2}$, associated to a force $\vec{f}$ and if $\vec{u}$ and $\vec{f}$ satisfy the following assumptions 
- $\vec{u}$ belongs to $L_{t}^{\infty} L_{x}^{2} \cap L_{t}^{2} H_{x}^{1}$,

- $\operatorname{div} \vec{f}=0$ and $\vec{f} \in L_{t}^{2} H_{x}^{k}\left(\Omega_{2}\right)$ for some $k \in \mathbb{N}$,

- $\vec{u} \in \mathcal{M}^{s, \tau}\left(\Omega_{2}\right)$ with $\tau>5$ and $2<s \leq \tau$,

then, for every subdomain $\Omega_{3}$ which is relatively compact in $\Omega_{2}$, we have

$$
\vec{u} \in L_{t}^{\infty} H_{x}^{k+1} \cap L_{t}^{2} H_{x}^{k+2}\left(\Omega_{3}\right) .
$$

Using those theorems, Chamorro, Lemarié-Rieusset and Mayoufi [Ch] could prove the following theorem (which is essentially the result proved previously by Wolf $[\mathbf{W}]$ ) :

Theorem 4.8 (Wolf's theorem). Let $\Omega$ be a domain in $\mathbb{R} \times \mathbb{R}^{3}, \vec{f} \in L_{\text {loc }}^{2}(\Omega)$ a divergence-free time-dependent vector field and $\vec{u}$ a local weak solution of the Navier-Stokes equations on $\Omega$ (associated to the force $\vec{f}$ ). Assume that moreover

- $\vec{u}$ is dissipative,

- the force $\vec{f}$ is regular : $\vec{f}$ belongs locally to $L_{t}^{2} H_{x}^{1}$,

Then:

- if $(t, x) \notin \Sigma(\vec{u})$, then

$$
\lim _{r \rightarrow 0} \frac{1}{r} \iint_{Q_{r}(t, x)}|\vec{\nabla} \otimes \vec{u}|^{2} d s d y=0 .
$$

- if $(t, x) \in \Sigma(\vec{u})$, then

$$
\limsup _{r \rightarrow 0} \frac{1}{r} \iint_{Q_{r}(t, x)}|\vec{\nabla} \otimes \vec{u}|^{2} d s d y \geq \epsilon^{*}
$$

where $\epsilon^{*}$ is a positive constant (which doesn't depend on $\vec{u}, \vec{f}$ nor $\Omega$ ).

Proof. We sketch the proof given in $\left[\mathbf{C h}, \mathbf{L e}\right.$. Let $\epsilon^{*}$ be the constant in Kukavica's theorem. Let $\left(x_{0}, t_{0}\right) \in \Omega$ with

$$
\limsup _{r \rightarrow 0} \frac{1}{r} \iint_{Q_{r}\left(t_{0}, x_{0}\right)}|\vec{\nabla} \otimes \vec{u}|^{2} d s d y<\epsilon^{*} .
$$

We introduce a harmonic correction $\vec{H}$ on a cylindric neighborhood of $\left(x_{0}, t_{0}\right)$ and consider the vector field $\vec{U}=\vec{u}+\vec{H}$. If $\vec{u}$ is dissipative, then $\vec{U}$ is suitable, associated to a force $\vec{F} \in L_{t}^{2} L_{x}^{2}(Q)$ and a pressure $P \in L_{t}^{3 / 2} L_{x}^{3 / 2}(Q)$. Moreover,

$$
\limsup _{r \rightarrow 0} \frac{1}{r} \iint_{Q_{r}\left(t_{0}, x_{0}\right)}|\vec{\nabla} \otimes \vec{U}|^{2} d s d y=\limsup _{r \rightarrow 0} \frac{1}{r} \iint_{Q_{r}\left(t_{0}, x_{0}\right)}|\vec{\nabla} \otimes \vec{u}|^{2} d s d y<\epsilon^{*} .
$$

Thus, by Kukavica's theorem, there exists $\tau>5$ and a neighborhood $\Omega_{2} \subset Q$ of $\left(t_{0}, x_{0}\right)$ such that $\vec{U} \in \mathcal{M}^{3, \tau}\left(\Omega_{2}\right)$. As $\vec{u}=\vec{U}-\vec{H}$, we see that we have as well $\vec{u} \in \mathcal{M}^{3, \tau}\left(\Omega_{2}\right)$. As $\vec{f} \in L_{t}^{2} H_{x}^{1}$, we may use O'Leary's theorem and find that, on a cylindric neighborhood $\Omega_{3}$ of $\left(t_{0}, x_{0}\right)$, we have $\vec{u} \in L_{t}^{\infty} H_{x}^{2}\left(\Omega_{3}\right) \subset L_{t, x}^{\infty}\left(\Omega_{3}\right)$. Thus, $\left(t_{0}, x_{0}\right) \notin \Sigma(\vec{u})$.

\section{Weak convergence of local weak solutions.}

In this final section, we prove Theorem 1.3. Recall that we consider a sequence $\left(\vec{f}_{n}\right)_{n \in \mathbb{N}}$ of divergence-free time-dependent vector fields on a domain $\Omega \subset \mathbb{R} \times \mathbb{R}^{3}$ and a sequence $\left(\vec{u}_{n}\right)_{n \in \mathbb{N}}$ of local weak solutions of the Navier-Stokes equations on $\Omega$ (associated to the forces $\vec{f}_{n}$ ) such that, for each cylinder $Q \subset \subset \Omega$, we have 
- $\vec{f}_{n} \in L_{t}^{2} H_{x}^{1}(Q)$ and $\vec{f}_{n}$ converges weakly in $L_{t}^{2} H_{x}^{1}$ to a limit $\vec{f}$,

- the sequence $\vec{u}_{n}$ is bounded in $L_{t}^{\infty} L_{x}^{2}(Q) \cap L_{t}^{2} H_{x}^{1}(Q)$ and converges weakly in $L_{t}^{2} H_{x}^{1}(Q)$ to a limit $\vec{u}$,

- for every $n, \vec{u}_{n}$ is bounded on $Q$ (the bound depending on $n$ ).

We know that we may define a pressure $p_{n}$ on $Q$ and that we have the energy equality

$$
M\left(\vec{u}_{n}\right)=0,
$$

where

$$
\begin{aligned}
M\left(\vec{u}_{n}\right)= & -\partial_{t}\left|\vec{u}_{n}\right|^{2}+\Delta\left(\left|\vec{u}_{n}\right|^{2}\right)-2\left|\vec{\nabla} \otimes \vec{u}_{n}\right|^{2}-\operatorname{div}\left(\left|\vec{u}_{n}\right|^{2} \vec{u}_{n}\right) \\
& -2<<\operatorname{div}\left(p_{n} \vec{u}_{n}\right)>>+2 \vec{u}_{n} \cdot \vec{f}_{n} .
\end{aligned}
$$

Our aim is then to prove that the limit $\vec{u}$ is a solution to the Navier-Stokes aquations associated to the limit $\vec{f}$ and that this solution is dissipative :

$$
M(\vec{u}) \geq 0 .
$$

We cannot give a direct proof, as it is possible that no term in the definition of $M\left(\vec{u}_{n}\right)$ converge to the corresponding term in $M(\vec{u}): p$ is not the limit in $\mathcal{D}^{\prime}$ of $p_{n}$ and $|\vec{u}|^{2}$ is not the limit in $\mathcal{D}^{\prime}$ of $\left|\vec{u}_{n}\right|^{2} \ldots$ It is easy to find an example of such a bad behavior by studying Serrin's example of smooth in space and singular in time solution to the Navier-Stokes equations [Se] :

EXAMPLE 5.1 (Serrin's example). Let $\psi$ be defined on a neighborhood of $B\left(x_{0}, r_{0}\right)$ and be harmonic, $\Delta \psi=0$, and let $\vec{f}=0$ and

$$
\vec{u}=\alpha(t) \vec{\nabla} \psi(x)
$$

where $\alpha \in L^{\infty}((a, b))$. Then $\vec{u}$ is a local weak solution of the Navier-Stokes equations on $(a, b) \times B\left(x_{0}, r_{0}\right)$ :

$$
\partial_{t} \vec{u}=\Delta \vec{u}-\vec{u} \cdot \vec{\nabla} \vec{u}-\vec{\nabla}\left(-\dot{\alpha} \psi-\frac{|\vec{u}|^{2}}{2}\right)+\vec{f} .
$$

Clearly, if $\alpha$ is not regular, the pressure $p$ has no integrability in the time variable (because of the presence of the singular term $\dot{\alpha}(t)$ )and $\vec{u}$ has no regularity in the time variable. Thus, $\vec{u}$ is dissipative (as a matter of fact, $M(\vec{u})=0$ ) but not suitable, as it violates both assumptions and conclusions of the Caffarelli, Kohn and Nirenberg theorem.

Let us adapt this example to our problem. We define

$$
\vec{u}_{n}(t, x)=\cos (n t)\left(\begin{array}{c}
x_{1} \\
-x_{2} \\
0
\end{array}\right)
$$

- $\vec{u}_{n}$ is a solution on $\mathbb{R} \times \mathbb{R}^{3}$ of

$$
\left\{\begin{aligned}
\partial_{t} \vec{u}_{n} & =\Delta \vec{u}_{n}-\left(\vec{u}_{n} \cdot \vec{\nabla}\right) \vec{u}_{n}-\vec{\nabla} p_{n} \\
\operatorname{div} \vec{u}_{n} & =0
\end{aligned}\right.
$$

- In this example, we have for a bounded domain $\Omega_{0}$

$$
\vec{u}_{n} \rightarrow 0
$$


10 DIEGO CHAMORRO, PIERRE GILLES LEMARIÉ-RIEUSSET, AND KAWTHER MAYOUFI

in $L_{t}^{2} H_{x}^{1}\left(\Omega_{0}\right)$ and

$$
\left(\vec{u}_{n} \cdot \vec{\nabla}\right) \vec{u}_{n}-\frac{1}{2}\left(\begin{array}{c}
x_{1} \\
x_{2} \\
0
\end{array}\right) \neq 0
$$

in $\mathcal{D}^{\prime}\left(\Omega_{0}\right)$.

In order to circumvene this problem of non-convergence, we shall use two tools : equations on vorticities $\vec{\omega}_{n}=\vec{\nabla} \wedge \vec{u}_{n}$ and on harmonic corrections $\vec{U}_{n}=\vec{u}_{n}+\vec{H}_{n}=$ $-\frac{1}{\Delta} \vec{\nabla} \wedge\left(\psi\left(\vec{\nabla} \wedge \vec{u}_{n}\right)\right)$.

\section{Step 1 : Vorticities.}

On a cylinder $Q \subset \subset \Omega$, we may write the Navier-Stokes equations on the divergence-free vector field $\vec{u}_{n}$ in many ways. The first one is given by equation (1) : for every smooth compactly supported divergence-free vector field $\vec{\phi} \in \mathcal{D}(Q)$ we have

$$
\iint_{Q} \vec{u}_{n} \cdot\left(\partial_{t} \vec{\phi}+\Delta \vec{\phi}\right)+\vec{u}_{n} \cdot\left(\vec{u}_{n} \cdot \vec{\nabla} \vec{\phi}\right)+\vec{f}_{n} \cdot \vec{\phi} d t d x=0 .
$$

We may rewrite this equation as:

$$
\partial_{t} \vec{u}_{n}=\Delta \vec{u}_{n}-\vec{u}_{n} \cdot \vec{\nabla} \vec{u}_{n}+\vec{f}_{n} \text { in }\left(\mathcal{D}_{\sigma}(Q)\right)^{\prime}
$$

where $\mathcal{D}_{\sigma}(Q)$ is the space of smooth compactly supported divergence-free vector fields on $Q$.

The second one is given by equations (2): for a distribution $p_{n} \in \mathcal{D}^{\prime}(Q)$, we have

$$
\partial_{t} \vec{u}_{n}=\Delta \vec{u}_{n}-\vec{u}_{n} \cdot \vec{\nabla} \vec{u}_{n}-\vec{\nabla} p_{n}+\vec{f}_{n} \quad \text { in } \mathcal{D}^{\prime}(Q) .
$$

The next one is based on the identity

$$
\vec{u}_{n} \cdot \vec{\nabla} \vec{u}_{n}=\vec{\omega}_{n} \wedge \vec{u}_{n}+\vec{\nabla}\left(\frac{\left|\vec{u}_{n}\right|^{2}}{2}\right)
$$

from which we get

$$
\partial_{t} \vec{u}_{n}=\Delta \vec{u}_{n}-\vec{\omega}_{n} \wedge \vec{u}_{n}+\vec{f}_{n} \text { in }\left(\mathcal{D}_{\sigma}(Q)\right)^{\prime} .
$$

We have seen that, in some cases, we don't have the convergence of $\left(\vec{u}_{n} \cdot \vec{\nabla}\right) \vec{u}_{n}$ to $\vec{u} \cdot \vec{\nabla} \vec{u}$ in $\mathcal{D}^{\prime}\left(\Omega_{0}\right)$. But we shall prove the following lemma :

LEMMA 5.2 (Convergence of the non-linear term). We have the following convergence results :

$$
\vec{\omega}_{n} \wedge \vec{u}_{n} \rightarrow \vec{\omega} \wedge \vec{u} \text { in } \mathcal{D}^{\prime}(Q)
$$

so that

$$
\left(\vec{u}_{n} \cdot \vec{\nabla}\right) \vec{u}_{n} \rightarrow \vec{u} \cdot \vec{\nabla} \vec{u} \text { in }\left(\mathcal{D}_{\sigma}(Q)\right)^{\prime} .
$$

Thus, this lemma will prove the first half of Theorem 1.3 the limit $\vec{u}$ is a local weak solution on $\Omega$ of the Navier-Stokes equations associated to the force $\vec{f}$. The proof of the lemma is based on the following variant of the classical Rellich lemma [Le, M] :

LEMMA 5.3 (Rellich's lemma). Let $-\infty<\sigma_{1}<\sigma_{2}<+\infty$. Let $\Omega$ be a domain in $\mathbb{R} \times \mathbb{R}^{3}$. If a sequence of distribution $T_{n}$ is weakly convergent to a distribution $T$ in $\left(L_{t}^{2} H_{x}^{\sigma_{2}}\right)_{\mathrm{loc}}$ and if the sequence $\left(\partial_{t} T_{n}\right)$ is bounded in $\left(L_{t}^{2} H_{x}^{\sigma_{1}}\right)_{\mathrm{loc}}$, then $T_{n}$ is strongly convergent in $\left(L_{t}^{2} H_{x}^{\sigma}\right)_{\text {loc }}$ for every $\sigma<\sigma_{2}$. 
We apply Rellich's lemma to $\vec{\omega}_{n}$. We have

$$
\partial_{t} \vec{\omega}_{n}=\Delta \vec{\omega}_{n}-\operatorname{div}\left(\vec{u}_{n} \otimes \vec{\omega}_{n}-\vec{\omega}_{n} \otimes \vec{u}_{n}\right)-\vec{\nabla} \wedge \vec{f}_{n}
$$

so that the sequence $\left(\partial_{t} \vec{\omega}_{n}\right)$ is bounded in $\left(L_{t}^{2} H_{x}^{\sigma_{1}}\right)_{\text {loc }}$ for all $\sigma_{1}<-5 / 2$. Moreover, $\vec{\omega}_{n}$ is weakly convergent to $\vec{\omega}$ in $\left(L_{t}^{2} L_{x}^{2}\right)_{\text {loc }}$. Thus, $\vec{\omega}_{n}$ is strongly convergent in $\left(L_{t}^{2} H_{x}^{-1}\right)_{\text {loc }}$. As $\vec{u}_{n}$ is weakly convergent to $\vec{u}$ in $\left(L_{t}^{2} H_{x}^{1}\right)_{\text {loc }}$, we find that $\vec{\omega}_{n} \wedge \vec{u}_{n}$ is weakly convergent to $\vec{\omega} \wedge \vec{u}$ in $\mathcal{D}^{\prime}(\Omega)$.

\section{Step 2 : Harmonic corrections.}

We now end the proof of Theorem 1.3 by checking the dissipativity of the limit $\vec{u}$. We restate the theorem as a result of stability for dissipativity :

Theorem 5.4 (Dissipative limits). Let $\Omega$ be a domain in $\mathbb{R} \times \mathbb{R}^{3}$. Assume that we have sequences $\vec{f}_{n}$ of divergence-free time-dependent vector fields and $\vec{u}_{n}$ of local weak solutions of the Navier-Stokes equations on $\Omega$ (associated to the forces $\left.\vec{f}_{n}\right)$ such that, for each cylinder $Q \subset \subset \Omega$, we have

- $\vec{f}_{n} \in L_{t}^{2} H_{x}^{1}(Q)$ and $\vec{f}_{n}$ converges weakly in $L_{t}^{2} H_{x}^{1}$ to a limit $\vec{f}$,

- the sequence $\vec{u}_{n}$ is bounded in $L_{t}^{\infty} L_{x}^{2}(Q) \cap L_{t}^{2} H_{x}^{1}(Q)$ and converges weakly in $L_{t}^{2} H_{x}^{1}(Q)$ to a limit $\vec{u}$,

- for every $n, \vec{u}_{n}$ is dissipative.

Then the limit $\vec{u}$ is a dissipative local weak solution on $\Omega$ of the Navier-Stokes equations associated to the force $\vec{f}$.

Proof. We already know that $\vec{u}$ is a local weak solution on $\Omega$ of the NavierStokes equations associated to the force $\vec{f}$. We have to prove its dissipativity.

Let $Q \subset \subset \Omega$ be a cylinder and $\psi \in \mathcal{D}(\Omega)$ be a cut-off function which is equal to 1 on a neighborhood of $Q$. In order to prove that $\vec{u}$ is dissipative, we shall prove that the harmonic correction $\vec{U}=\vec{H}+\vec{u}=-\frac{1}{\Delta} \vec{\nabla} \wedge(\psi(\vec{\nabla} \wedge \vec{u}))$ is suitable.

We define as well $\vec{U}_{n}=-\frac{1}{\Delta} \vec{\nabla} \wedge\left(\psi\left(\vec{\nabla} \wedge \vec{u}_{n}\right)\right)$. The weak convergence of $\vec{u}_{n}$ in $\left(L_{t}^{2} H_{x}^{1}\right)_{\text {loc }}(\Omega)$ implies the weak convergence of $\vec{U}_{n}$ to $\vec{U}$ in $L_{t}^{2} H_{x}^{1}(Q)$. Moreover, the uniform boundedness of the sequence $\left(\vec{u}_{n}\right)_{n \in \mathbb{N}}$ in $\left(L_{t}^{2} H_{x}^{1} \cap L_{t}^{\infty} L_{x}^{2}\right)_{\text {loc }}(\Omega)$ and of the sequence $\left(\vec{f}_{n}\right)_{n \in \mathbb{N}}$ in $\left(L_{t}^{2} H_{x}^{1}\right)_{\text {loc }}(\Omega)$ implies that the sequences of pressure $P_{n}$ and of forces $\vec{F}_{n}$ associated to $\vec{U}_{n}$ are uniformly bounded (respectively in $L_{t}^{3 / 2} L_{x}^{3 / 2}(Q) \cap$ $L_{t}^{2} L_{x}^{6 / 5}(Q)$ and in $\left.L_{t}^{2} L_{x}^{2}(Q)\right)$. Thus, $\partial_{t} \vec{U}_{n}$ is bounded in $L_{t}^{2} H_{x}^{-2}(Q)$ and Rellich's lemma gives us that $\vec{U}_{n}$ is strongly convergent to $\vec{U}$ in $\left(L_{t}^{2} L_{x}^{2}\right)_{\text {loc }}(Q)$ (and, since $\vec{U}_{n}$ is bounded in $L_{t}^{10 / 3} L_{x}^{10 / 3}(Q)$, we have strong convergence in $\left(L_{t}^{3} L_{x}^{3}\right)_{\text {loc }}(Q)$ as well).

Taking subsequences, we may assume that the bounded sequences $P_{n}$ (in $L_{t}^{3 / 2} L_{x}^{3 / 2}(Q)$ ), $\vec{F}_{n}$ (in $\left.L_{t}^{2} L_{x}^{2}(Q)\right)$ and $\left|\vec{\nabla} U_{n}\right|^{2}$ (in $\left.L_{t}^{1} L_{x}^{1}(Q)\right)$ converge weakly in $\mathcal{D}^{\prime}$ to limits $P_{\infty} \in L_{t}^{3 / 2} L_{x}^{3 / 2}(Q), \vec{F}_{\infty} \in L_{t}^{2} L_{x}^{2}(Q)$ and $\nu_{\infty}$ (a non-negative finite measure on $Q$ ). In particular, we have enough convergence to see that every term in the right-hand side of equality

$M\left(\vec{u}_{n}\right)=-\partial_{t}\left|\vec{U}_{n}\right|^{2}+\Delta\left(\left|\vec{U}_{n}\right|^{2}\right)-2\left|\vec{\nabla} \otimes \vec{U}_{n}\right|^{2}-\operatorname{div}\left(\left|\vec{U}_{n}\right|^{2} \vec{U}_{n}\right)-2 \operatorname{div}\left(P_{n} \vec{U}_{n}\right)+2 \vec{U}_{n} \cdot \vec{U}_{n}$ has a limit, so that $\nu_{1}=\lim _{n \rightarrow+\infty} M\left(\vec{U}_{n}\right)$ exists and

$$
\nu_{1}=-\partial_{t}|\vec{U}|^{2}+\Delta\left(|\vec{U}|^{2}\right)-2 \nu_{\infty}-\operatorname{div}\left(|\vec{U}|^{2} \vec{U}\right)-2 \operatorname{div}\left(P_{\infty} \vec{U}\right)+2 \vec{U} \cdot \vec{F}_{\infty}
$$


As $M\left(\vec{U}_{n}\right) \geq 0$, we find that $\nu_{1} \geq 0$. Moreover, by the Banach-Steinhaus theorem, we find that $\nu_{2}=\nu_{\infty}-|\vec{\nabla} \otimes \vec{U}|^{2} \geq 0$. As $M(\vec{U})=\nu_{1}+2 \nu_{2}$, we have $M(\vec{U}) \geq 0$. Hence, $\vec{U}$ is suitable and $\vec{u}$ is dissipative.

\section{References}

[Ca] L. Caffarelli, R. Kohn \& L. Nirenberg. Partial regularity of suitable weak solutions of the Navier-Stokes equations. Comm. Pure Appl. Math., 35 (1982), 771-831.

[Ch] D. Chamorro, PG. Lemarié-Rieusset \& K. Mayoufi. The role of the pressure in the partial regularity theory for weak solutions of the Navier-Stokes equations. https://arxiv.org/abs/1602.06137, (2016).

[D] J. Duchon \& R. Robert. Inertial energy dissipation for weak solutions of incompressible Euler and Navier-Stokes equations. Nonlinearity, 13 (2000), 249-255.

[K] I. Kukavica. On partial regularity for the Navier-Stokes equations. Discrete and continuous dynamical systems, 21 (2008), 717-728.

[La] O. Ladyzhenskaya \& G. Seregin. On partial regularity of suitable weak solutions to the threedimension Navier-Stokes equations. J. Math. Fluid Mech., 1 (1999), 356-387.

[Le] P.G. Lemarié-Rieusset. The Navier-Stokes problem in the XXIst century, Chapman \& Hall/CRC, (2016).

$[\mathrm{M}]$ K. Mayoufi. Les inégalités d'énergie locales dans la théorie des équations de Navier-Stokes, Ph. D. Univ. Évry, (2017).

[O] M. OLeary. Conditions for the local boundedness of solutions of the Navier-Stokes system in three dimensions. Comm. Partial Differential Equations, 28 (2003), 617-636.

[Sc] V. Scheffer. Hausdorff measure and the Navier-Stokes equations. Comm. Math. Phys., 55 (1977), 97-112.

[Se] J. Serrin. On the interior regularity of weak solutions of the Navier-Stokes equations. Arch. Rat. Mech. Anal., 9 (1962), 187-195.

[W] J. Wolf. A direct proof of the Caffarelli-Kohn-Nirenberg theorem. Parabolic and NavierStokes equations. Part 2, 533-552, Banach Center Publ., 81, Part 2, Polish Acad. Sci. Inst. Math., Warsaw, 2008.

LAMme, Univ Evry, CNRS, Université Paris-Saclay, 91025, Evry, France

Current address: IBGBI, 23 bd de France, 91037 Évry cedex, France

E-mail address: diego.chamorro@univ-evry.fr

LAMme, Univ Evry, CNRS, Université Paris-Saclay, 91025, Evry, France

Current address: IBGBI, 23 bd de France, 91037 Évry cedex, France

E-mail address: pierregilles.lemarierieusset@univ-evry.fr

LAMme, Univ Evry, CNRS, Université Paris-Saclay, 91025, Evry, France

Current address: IBGBI, 23 bd de France, 91037 Évry cedex, France

E-mail address: kawther.mayoufi@univ-evry.fr 